\title{
Effect of Nanosilica and Titania on Thermal Stability of Polypropylene/Oil Palm Empty Fruit Fibre Composite.
}

\begin{abstract}
Degradation of polypropylene (PP) composites at elevated temperature for prolonged period has shortened the lifetime of PP composites. Thus, variety of fillers has been incorporated into PP matrix to improve thermal degradation stability. The effects of titania and nanosilica in PP reinforced with oil palm empty fruit bunch fibres (EFB) were investigated in this study. Mechanical properties of the samples were determined before and after thermal ageing. Morphology of the composite with varies fillers composition were analyzed using scanning electron microscope. The introduction of nanosilica into PP/EFB composite filled with titania has increased both the melting and glass transition temperature of PP. Chain splitting in molecular chains reduced with the incorporation of nanosilica and titania into PP and PP/FEB composites. The TGA study has showed that the addition of nanosilica has further enhanced the thermal stability effect of titania in PP/EFB composite. However, both the Izod impact strength and tensile strength of the composite reduced greatly after 20 days of thermal ageing.
\end{abstract}

Keyword: Composite; Nanoalumina; Titanium oxide; Polypylene 